\title{
71. INVESTIGATION OF THE ORBITAL STABILITY OF MINOR PLANETS WITH COMETARY ECCENTRICITIES
}

\author{
G. A. CHEBOTAREV, N. A. BELYAEV and R. P. EREMENKO \\ Institute for Theoretical Astronomy, Leningrad, U.S.S.R.
}

\begin{abstract}
The evolution of the orbits of 19 asteroids of particular interest has been studied over the interval 1660-2060, perturbations by Venus to Pluto being taken into account. Information was obtained about the encounters with Venus, the Earth, and Mars. A few approaches of Hidalgo to Jupiter were noted. In distinction to the orbits of short-period comets, the orbits of the 19 asteroids are stable throughout the 400 -yr interval.
\end{abstract}

\section{The Group of Minor Planets with 'Cometary' Eccentricities}

Among the known minor planets the group with so-called 'cometary' eccentricities is of major interest. The orbits of this group deserve to be thoroughly investigated in view of the possible genetic relationship between minor planets and comets, for these are the objects that might be 'former comets', i.e., cometary nuclei lacking comas, a problem that is being widely discussed nowadays (Gehrels et al., 1970; Kresák, 1969; Marsden, 1970).

Another reason for studying this 'cometary' group of minor planets is that there may be close approaches to the Earth and other inner planets. Hermes, for instance, has approached the Earth to within $0.005 \mathrm{AU}$, and Apollo was only $0.00056 \mathrm{AU}$ $(85000 \mathrm{~km})$ from Venus in March 1858 . The 19 minor planets studied in this work and their orbital elements are included in Table I in order of increasing mean daily motion $\mu$. Most of the elements are taken from the 1967 edition of Efemeridy Malykh Planet. The orbits of the lost asteroids, indicated by asterisks, are based on observations at one opposition only and are therefore unreliable.

The structure of the belt of minor planets is characterized by the data shown in Table II (Chebotarev and Shmakova, 1970). Eight of the 19 planets under consideration here are in ring 2, one planet is in ring 3, and ten planets are outside the main belt, one of them being between the orbits of Jupiter and Saturn. The mean eccentricity $e$ for the group is 0.50 , as opposed to 0.15 for the whole system of minor planets.

Owing to the large eccentricities of their orbits, some of the asteroids pass inside the orbits of Mars, the Earth, and Venus and may have close encounters with these major planets; but the aphelion distances show that close approaches to Jupiter are impossible. The inclinations $i$ are another distinct feature of the orbits of this group of minor planets, varying from $1: 5$ (Adonis) to $52^{\circ}$ (Betulia); the mean value of 17.4 is almost twice the mean inclination (9.5) for the majority of the minor planets.

Some of the short-period comets of Jupiter's family have orbits similar to those of minor planets with 'cometary' eccentricities; see Table III. Comparison of Tables I and III shows that the cometary orbits really are different, having large eccentricities and comparatively low inclinations. Of the 19 asteroids only three (Hidalgo, Adonis and Icarus), strictly speaking, have typical cometary orbits. It is interesting to note that 
G. A. Chebotarev et Al.

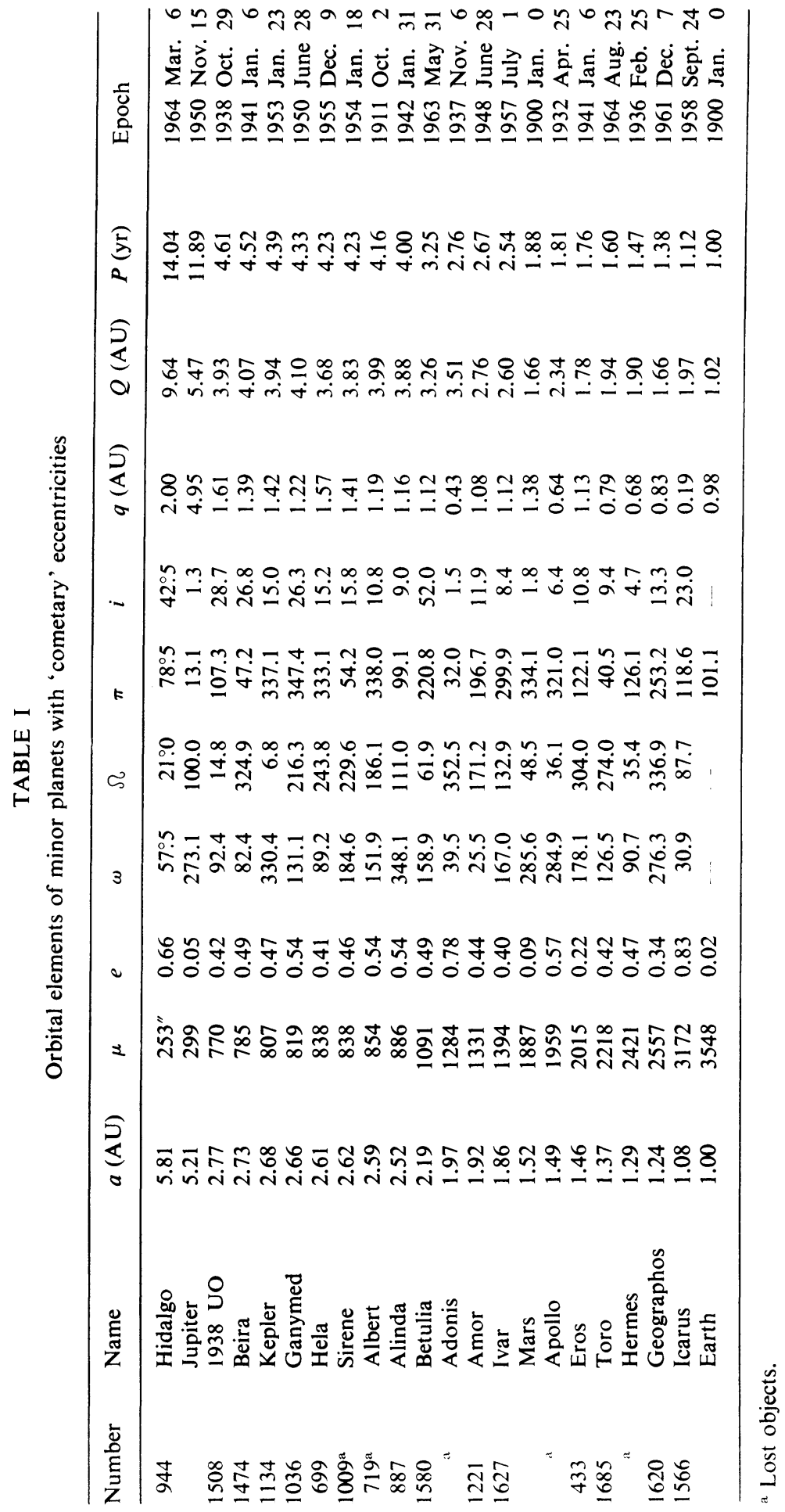


TABLE II

The minor planet belt

\begin{tabular}{llrlrl}
\hline & Interval of $\mu$ & $\Delta \mu$ & Commensurability & $N$ & $a$ (AU) \\
\hline Ring 1 & $610^{\prime \prime}-740^{\prime \prime}$ & 130 & - & 659 & $3.23-2.84$ \\
Gap & $740-750$ & 10 & $2: 5$ & 4 & $2.84-2.82$ \\
Ring 2 & $750-890$ & 140 & - & 537 & $2.82-2.51$ \\
Gap & $890-910$ & 20 & $1: 3$ & 7 & $2.51-2.48$ \\
Ring 3 & $910-1110$ & 200 & - & 420 & $2.48-2.17$ \\
Total & $610-1110$ & & & 1627 & $3.23-2.17$ \\
\hline
\end{tabular}

$N$ gives the number of minor planets within the regions specified.

the orbit of Adonis resembles that of Encke's Comet. On the other hand, P/WilsonHarrington has a typical asteroidal orbit similar to that of the planet 1627 Ivar.

The shapes and sizes of the asteroids play an important role in solving the problem of relationship between comets and minor planets. The latest investigations show Icarus to be nearly spherical (radius $0.54 \mathrm{~km}$ ), while Eros and Geographos, for instance, are distinctly irregular in form (Eros being 35 by 16 by $7 \mathrm{~km}$; Geographos 2.4 by $0.7 \mathrm{~km}$ ).

\section{Evolution of the Orbits of Minor Planets over $400 \mathrm{yr}$}

The orbital evolution of the 19 asteroids is shown in Table IV, which gives the extremes in the orbital elements between 1660 and 2060. The equations of motion have been integrated on an electronic computer by Cowell's method with automatic variation of the integration step and allowance being made for perturbations by Venus to Pluto (Belyaev, 1967).

The principal result obtained is that the planetary orbits with cometary eccentricities are exceptionally stable compared with the orbits of typical short-period comets, the latter being characterized by close approaches to the major planets and drastic changes in the elements.

The greatest perturbations in longitude of perihelion are exhibited by 1009 Sirene $(\Delta \pi=6: 0)$ and 944 Hidalgo $(\Delta \pi=-5: 9)$, while 1566 Icarus and Apollo $(\Delta \pi= \pm 0.2)$ show the smallest. The planets of the Hilda and Thule groups are quite different (Chebotarev et al., 1970), the line of apsides of 1269 Rollandia moving through $\Delta \pi=156: 9$ and that of 279 Thule through $\Delta \pi=-335^{\circ}: 3$ during the same $400-y r$ interval.

The perturbations in inclination are extremely small $(\Delta i= \pm 2: 5)$, except that for 1474 Beira $\Delta i=-8.8$. The eccentricity variations are also extremely small $\left(\Delta e_{\max }=\right.$ 0.05 ). As a consequence of the minor variations in semimajor axis and eccentricity the perihelion and aphelion distances also change insignificantly, illustrating the longterm stability of the orbits. The maximum changes in perihelion distance are observed for 1627 Ivar $(\Delta q=0.14)$ and 944 Hidalgo $(\Delta q=0.16)$.

The motion of planet 887 Alinda is of special interest (Table V). The mean motion librates about the 1:3 commensurability with Jupiter $\left(\mu^{*}=897^{\prime \prime}\right)$. During the $400-\mathrm{yr}$ 


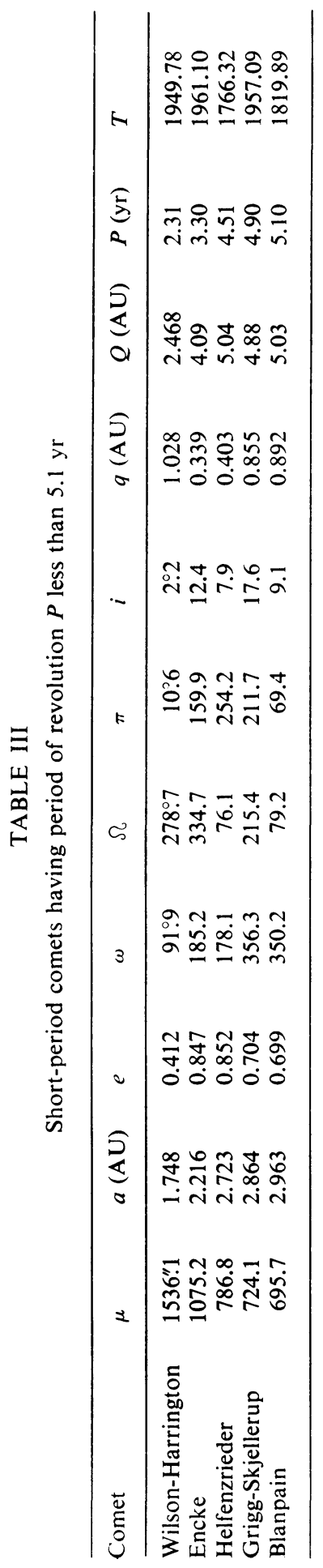


TABLE IV

Variations in the elements of minor planets over 1660-2059

\begin{tabular}{|c|c|c|c|c|c|c|c|c|c|c|}
\hline Planet & $a(\mathrm{AU}$ & & $e$ & $\omega$ & $\Omega$ & $\pi$ & $i$ & $q(\mathrm{AU})$ & $Q(\mathrm{AU})$ & $P(\mathrm{yr})$ \\
\hline \multirow[t]{2}{*}{699} & 2.61 & $840^{\prime \prime}$ & 0.41 & $79: 8$ & $252: 3$ & $332: 0$ & $15: 3$ & 1.55 & 3.67 & 4.22 \\
\hline & 2.61 & 839 & 0.41 & 93.5 & 240.3 & 337.7 & 15.3 & 1.55 & 3.67 & 4.23 \\
\hline \multirow[t]{2}{*}{719} & 2.59 & 853 & 0.55 & 140.5 & 194.2 & 334.7 & 9.4 & 1.17 & 4.01 & 4.16 \\
\hline & 2.58 & 858 & 0.54 & 156.2 & 182.9 & 339.1 & 11.5 & 1.18 & 3.98 & 4.14 \\
\hline \multirow[t]{2}{*}{887} & 2.48 & 910 & 0.56 & 347.1 & 113.3 & 100.4 & 9.1 & 1.09 & 3.87 & 3.90 \\
\hline & 2.47 & 916 & 0.58 & 350.6 & 109.5 & 100.0 & 9.5 & 1.05 & 3.89 & 3.87 \\
\hline \multirow[t]{2}{*}{944} & 5.67 & 263 & 0.63 & 58.6 & 24.1 & 82.7 & 44.9 & 2.12 & 9.22 & 13.49 \\
\hline & 5.82 & 253 & 0.66 & 56.4 & 20.3 & 76.8 & 42.4 & 1.98 & 9.66 & 14.03 \\
\hline \multirow[t]{2}{*}{1009} & 2.62 & 836 & 0.46 & 175.7 & 233.6 & 49.3 & 15.7 & 1.42 & 3.82 & 4.24 \\
\hline & 2.62 & 837 & 0.46 & 187.0 & 228.2 & 55.3 & 15.8 & 1.42 & 3.82 & 4.24 \\
\hline \multirow[t]{2}{*}{1036} & 2.66 & 817 & 0.56 & 124.4 & 223.5 & 347.9 & 24.5 & 1.18 & 4.15 & 4.34 \\
\hline & 2.66 & 816 & 0.53 & 133.5 & 213.9 & 347.4 & 27.0 & 1.25 & 4.08 & 4.35 \\
\hline \multirow[t]{2}{*}{1134} & 2.68 & 809 & 0.48 & 320.0 & 13.4 & 333.4 & 14.1 & 1.40 & 3.96 & 4.39 \\
\hline & 2.68 & 810 & 0.47 & 333.5 & 4.6 & 338.0 & 15.4 & 1.42 & 3.94 & 4.38 \\
\hline \multirow[t]{2}{*}{1221} & 1.93 & 1327 & 0.43 & 20.6 & 173.7 & 194.3 & 12.1 & 1.09 & 2.77 & 2.67 \\
\hline & 1.92 & 1334 & 0.44 & 27.4 & 170.3 & 197.7 & 11.9 & 1.08 & 2.76 & 2.66 \\
\hline \multirow[t]{2}{*}{1474} & 2.74 & 784 & 0.48 & 79.6 & 330.9 & 50.5 & 35.5 & 1.41 & 4.07 & 4.53 \\
\hline & 2.74 & 784 & 0.49 & 84.7 & 321.8 & 46.5 & 26.7 & 1.40 & 4.08 & 4.53 \\
\hline \multirow[t]{2}{*}{1508} & 2.77 & 771 & 0.42 & 88.4 & 21.0 & 109.4 & 28.6 & 1.60 & 3.94 & 4.60 \\
\hline & 2.77 & 769 & 0.42 & 93.4 & 12.6 & 106.0 & 28.7 & 1.62 & 3.92 & 4.62 \\
\hline \multirow[t]{2}{*}{1566} & 1.08 & 3172 & 0.82 & 29.0 & 89.5 & 118.5 & 23.7 & 0.19 & 1.97 & 1.12 \\
\hline & 1.08 & 3172 & 0.83 & 31.7 & 87.0 & 118.7 & 22.7 & 0.19 & 1.97 & 1.12 \\
\hline \multirow[t]{2}{*}{1580} & 2.19 & 1092 & 0.52 & 156.2 & 63.3 & 219.5 & 51.5 & 1.06 & 3.33 & 3.25 \\
\hline & 2.19 & 1093 & 0.49 & 159.9 & 61.4 & 221.3 & 52.3 & 1.13 & 3.26 & 3.25 \\
\hline \multirow[t]{2}{*}{1620} & 1.24 & 2557 & 0.34 & 273.5 & 339.2 & 252.7 & 13.3 & 0.83 & 1.66 & 1.39 \\
\hline & 1.24 & 2555 & 0.34 & 277.2 & 336.2 & 253.4 & 13.3 & 0.83 & 1.66 & 1.39 \\
\hline \multirow[t]{2}{*}{1627} & 1.86 & 1393 & 0.40 & 163.2 & 134.8 & 298.0 & 8.3 & 1.12 & 2.60 & 2.55 \\
\hline & 1.86 & 1394 & 0.40 & 168.3 & 132.2 & 300.5 & 8.5 & 1.12 & 2.60 & 2.55 \\
\hline \multirow[t]{2}{*}{1685} & 1.37 & 2216 & 0.42 & 123.3 & 276.8 & 39.9 & 9.3 & 0.79 & 1.95 & 1.60 \\
\hline & 1.37 & 2216 & 0.42 & 127.7 & 273.2 & 40.9 & 9.4 & 0.79 & 1.95 & 1.60 \\
\hline \multirow[t]{2}{*}{ Apollo } & 1.53 & 1872 & 0.58 & 277.9 & 43.7 & 321.4 & 5.8 & 0.65 & 2.42 & 1.90 \\
\hline & 1.49 & 1956 & 0.57 & 286.8 & 34.5 & 321.2 & 6.4 & 0.64 & 2.33 & 1.81 \\
\hline \multirow[t]{2}{*}{ Hermes } & 1.30 & 2401 & 0.48 & 85.5 & 39.6 & 125.1 & 4.8 & 0.68 & 1.92 & 1.48 \\
\hline & 1.29 & 2413 & 0.48 & 94.5 & 31.9 & 126.3 & 4.5 & 0.68 & 1.91 & 1.47 \\
\hline \multirow[t]{2}{*}{ Adonis } & 1.97 & 1280 & 0.78 & 24.6 & 365.9 & 30.5 & 2.0 & 0.43 & 3.51 & 2.77 \\
\hline & 1.97 & 1280 & 0.78 & 48.5 & 344.1 & 32.6 & 1.3 & 0.44 & 3.51 & 2.77 \\
\hline
\end{tabular}

TABLE V

Orbital elements of minor planet 887 Alinda

\begin{tabular}{|c|c|c|c|c|c|c|c|c|c|c|}
\hline Epoch & $a(\mathrm{AU})$ & $\mu$ & $e$ & $\omega$ & $\Omega$ & $\pi$ & $i$ & $q(\mathrm{AU})$ & $Q(\mathrm{AU})$ & $P(\mathrm{yr})$ \\
\hline 1660 Feb. 8 & 2.48 & 910" & 0.56 & 347.1 & $113: 3$ & $100: 4$ & 9.1 & 1.09 & 3.87 & 3.90 \\
\hline 1700 Dec. 15 & 2.47 & 917 & 0.57 & 347.3 & 112.9 & 100.2 & 9.1 & 1.06 & 3.88 & 3.87 \\
\hline 1800 Jan. 25 & 2.50 & 895 & 0.55 & 347.7 & 112.2 & 99.9 & 9.0 & 1.13 & 3.87 & 3.96 \\
\hline 1900 Apr. 11 & 2.53 & 880 & 0.53 & 347.8 & 111.4 & 99.3 & 8.9 & 1.19 & 3.87 & 4.03 \\
\hline 1942 Jan. 31 & 2.52 & 886 & 0.54 & 348.1 & 111.0 & 99.1 & 9.0 & 1.16 & 3.88 & 4.00 \\
\hline 2000 Jan. 17 & 2.48 & 906 & 0.56 & 350.0 & 110.0 & 100.0 & 9.3 & 1.08 & 3.88 & 3.92 \\
\hline 2059 Dec. 13 & 2.47 & 916 & 0.58 & 350.6 & 109.5 & 100.0 & 9.5 & 1.05 & 3.89 & 3.87 \\
\hline
\end{tabular}


interval Alinda passes through the exact resonance twice. At the epochs 1660 and 1700 it is inside ring 3, and in 1800 it is inside the gap $\left(\mu=895^{\prime \prime}\right)$; between 1900 and 1942 Alinda is in ring 2, while around 2000 it enters the gap $(\mu=906 ")$ once more, returning to ring 3 in 2059. Since the variations in eccentricity compensate for those in semimajor axis the aphelion distance remains practically constant, and there is no change in the line of apsides either.

Considering the character of close encounters (less than $0.10 \mathrm{AU}$ ) with the Earth, Mars, and Venus, the 19 planets may be divided into the following groups:

(1) No close approaches $(433,699,799,1009,1036,1474,1508,1580,1627,1685)$.

(2) Close approaches to the Earth only $(887,1221,1620)$.

(3) Close approaches to Mars only (1134).

(4) Close approaches to Venus only (no planets).

(5) Close approaches to two planets (no planets).

(6) Close approaches to three planets (Adonis, Apollo, Hermes, 1566).

In addition there is 944 Hidalgo, which has four encounters with Jupiter (to less than 2 AU) between 1660 and 2060 (Table VI). These approaches did not appreciably change the orbit of Hidalgo.

TABLE VI

Encounters of 944 Hidalgo with Jupiter, 1660-2060

\begin{tabular}{lrll}
\hline \multicolumn{1}{l}{ Date } & & Minimum distance (AU) \\
\hline 1673 Aug. & 8 & 0.38 \\
1756 & Dec. & 1 & 1.51 \\
1827 & July & 3 & 0.84 \\
1922 & Oct. & 13 & 0.90 \\
\hline
\end{tabular}

\section{References}

Belyaev, N. A.: 1967, Byull. Inst. Teor. Astron. 10, 696.

Chebotarev, G. A., Belyaev, N. A., and Eremenko, R. P.: 1970, Byull. Inst. Teor. Astron. $12,82$.

Chebotarev, G. A. and Shmakova, M. Ya.: 1970, Byull. Inst. Teor. Astron. $12,104$.

Gehrels, T., Roemer, E., Taylor, R. C., and Zellner, B. H.: 1970, Astron. J. $75,186$.

Kresák, L.: 1969, Bull. Astron. Inst. Czech. 20, 177.

Marsden, B. G.: 1970, Astron. J. 75, 206. 